\title{
INTELLIGENT HOME HEATING SYSTEM
}

\author{
P.T Rajeena Mol ${ }^{1}$, B.Chinthamani ${ }^{2}$, K.P.Kamini ${ }^{3}$, K.R.Sugashini ${ }^{4}$ \\ 1,2 -Assistant Professor (Sl.Grade), ${ }^{3,4}$ Assistant Professor \\ Department of Electronics and Instrumentation Engineering, Easwari Engineering College, Ramapuram \\ Chennai - Tamilnadu 600089, India \\ rajeenamol2009@gmail.com,kaminipalani@yahoo.com
}

\begin{abstract}
To develop a home heating system with high efficiency, the outdoor temperature is not the only parameter to be considered but also disturbances such as ventilation, door/window openings and personal lifestyle. The aim of the project is to develop the home heating system which adapts itself to changing customer demands using fuzzy logic. The energy consumption and the outdoor temperature of the house were monitored over a period of one year to produce the data required to program the fuzzy controller and thereby the appropriate set temperature will be determined. When load disturbances such as door/window openings are given, there is an increase in the energy required by the heater. This additional energy is obtained as an output from the fuzzy controller and given to the heating system. The design, optimization and implementation of the fuzzy controller are supported by LabVIEW software
\end{abstract}

$* * *$

\section{INTRODUCTION}

A home heating system consists of a sensor installed at the outside of the house to measure the outdoor temperature. In order to meet the different needs of the customer, the room set temperature gets constantly adjusted in relation to the outdoor temperature, i.e. Tset $=\mathrm{f}$ (Toutdoor) as defined by European standards in the 1950s. The controller itself realizes an on-off characteristic. If the actual temperature inside room drops below the set temperature, system starts the heating process. Similarly, when actual temperature inside room goes above the set temperature, system stops the heating process.

Now a days most houses have good thermal insulation, therefore set temperature depend largely on the outdoor temperature. This set temperature is highly inappropriate. The existing method becomes obsolete owing to the improvement of house insulation. Also the existing method fails to consider several factors such as ventilation, door and window openings. The most important criteria describing individual customer heating demand patterns come from the actual energy consumption curve of the house, which is measured from the existing system. From the curve, these descriptive parameters are derived:

1. Current energy consumption, indicating current load

2. Medium-term tendency (I), indicating heating-up and heating-down phases,

3. Short-term tendency (II), indicating disturbances like door/window openings

4. Previous day's average energy consumption, indicating the house heating level

These four descriptive parameters are used to form rules heuristically for the determination of the appropriate set temperature. To allow for the formulation of plausibility rules the average outdoor temperature for every month is also a system input. The fuzzy controller uses a total of all these five inputs and provides an output which represents estimated heat requirement of the house.

The fuzzy controller is highly responsive to the actual heat requirement of the house as it considers several factors such as heater on/off, door/window opening etc and proves to be very reactive to any such sudden changes. Additionally, the elimination of the outdoor temperature sensor saves money and even more on installation costs. In case of low-load periods, the fuzzy controller saves a lot of energy. Also there is no mathematical model involved in the designing steps which happens to make the system simple to understand.

The system demonstrates acute response to even small changes in the input or the load conditions prevailing in the house. Since the need for mathematical model does not exist, it effectively reduces the complexity of the system. Hence our project not only reduces the cost substantially but also makes the system intelligent. This also has the major advantage over the conventional system in terms of economy and thermal comfort. It satisfies customer demands in all possible ways. This also happens to increase its commercial value. Thus, the intelligent home heating system is improved beyond the scope of a conventional heating system.

\section{MICROCONTROLLER AND CIRCUITS}

The hardware used in our project is as shown. Each component signifies different aspects in the centralized heating system. 


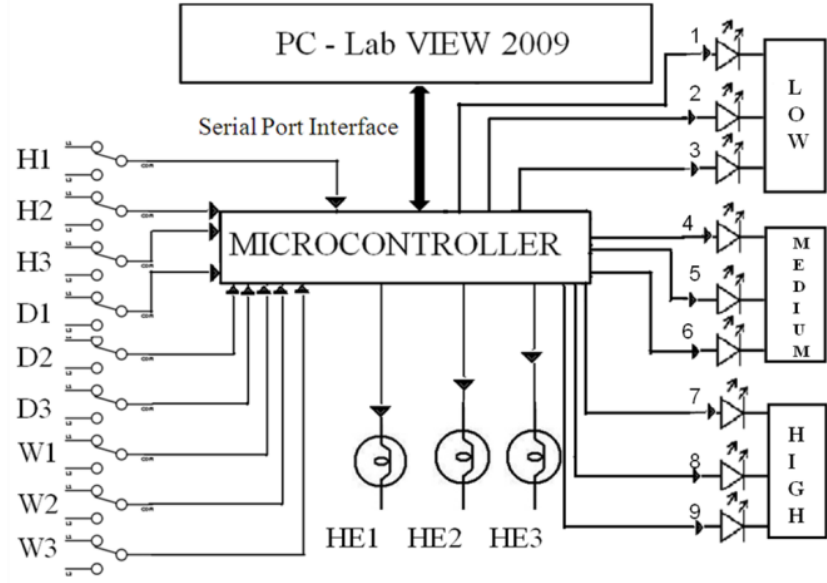

It consists of three bulbs to denote the heaters of the rooms in the centralized heating system. These bulbs are controlled by ON-OFF switches also attached in the hardware. The switch 1 indicates heater 1 , switch 2 indicates heater 2 and so on. The doors' opening or closing are denoted by switches 4,5 and 6 . Similarly the windows' opening and closing are denoted by switches 7,8 and 9.

The bulbs signify the heater load and in the same way the other switches represent the compensation required when any of the doors/ windows is left open.

There is a set of LED bulbs red, yellow and green which indicates the range of the thermal compensation being carried out at that instant. Red indicates 'high', yellow indicates 'medium' and green indicates 'low'. According to the changes in the lifestyle of people the thermal compensation will change. These are shown visually by the LED bulbs. The 9 bulbs symbolize the extent of compensation provided due to door/window opening/closing.

The interfacing part of the hardware with the software [LabVIEW] is executed through the USB to serial port converter. This will ensure that the signal is transmitted from the hardware and the controller obtains this as the input in the software. For example when the heater 1 is switched On, the controller in LabVIEW will get the input as logic 1 for heater 1 and it corresponds to the switching ON of heater in the front panel. The power supplied is denoted both as numerical data and as graphical data in the display.

The block diagram in the LabVIEW will indicate the logic of the controller. Fuzzy logic tool kit was used along with add-ons including VI manager 2010 which made it possible to make graphical developments and pictorial representations. Thus the sophisticated control was effectively done using these advanced tools in LabVIEW 2009.

\section{DEVELOPING FUZZY LOGIC CONTROLLER}

The objective of the fuzzy controller in our project is to estimate the additional heat which has to be added or subtracted based on the current load conditions.

The inputs to fuzzy logic controller were:

1)Average outside temperature

2)Medium term tendency

3)Short term tendency

The IF-THEN RULES were defined to obtain the ADDITIONAL HEAT INPUT.

The structure of the fuzzy controller is shown:

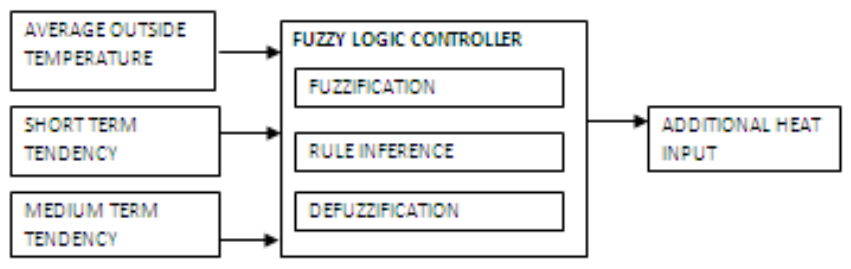

\section{MEMBERSHIP FUNCTIONS}

Outside temperature is the current outdoor temperature of the house for which the system is developed. This is recorded over a period of one year to get the temperature data base of that particular region. This is recorded in a data table as shown. The temperature which is average for each month in taken. For example January has an average of 17 degree Celsius and February has an average of 21 degree Celsius etc.

Since the data is pre-recorded, the need for outdoor temperature sensor is eliminated. When a particular month is chosen, the controller automatically calculates the required power to raise the temperature to suit human comfort. But since the outdoor temperature is not the only deciding factor for determining the heat or extra power required, we design the membership functions for a variety of other factors too.

Medium term tendency is defined as the the difference between the required heat input at time instants $\mathrm{t} 2$ and $\mathrm{t} 1$. Medium term tendency=heat input at $\mathrm{t} 2$ - heat input at $\mathrm{t} 1$. (for $\mathrm{t} 2>\mathrm{t} 1$ ) and vice versa. For example the heat required at the previous instant was $800 \mathrm{~W}$ and now at $\mathrm{t} 2$ it is $600 \mathrm{~W}$, then the difference between the two will be $200 \mathrm{~W}$ and that will be the medium term tendency for the controller to calculate the required power to produce the heat.

Short term tendency refers to the window or door opening which has an effect on the heating system. The extent to which it has an effect is determined by recording the energy consumption graph for a year and the curve indicates that extra energy needs to be supplied whenever a window or door is opened. The extra energy needed is called the "energy compensation' required. Hence short term tendency is taken as the difference between the heat compensation for door and window opening at time instants $\mathrm{t} 2$ and $\mathrm{t} 1$. Denoted as short 
term tendency $=$ heat compensation for door and window at $\mathrm{t} 2$ heat compensation for door and window $\mathrm{t} 1$. (for $\mathrm{t} 2>\mathrm{t} 1$ ) and vice versa. For each door heat compensation required is assumed to be $20 \mathrm{~W}$. For each window heat compensation required is assumed to be $10 \mathrm{~W}$ in the hardware.

Considering a home with three rooms, the energy required by the heaters are summed up and added to the energy compensation required due to short term tendency and finally this is given as the current energy consumption.

This is an important function since the difference between the current consumption and the previous should be known. For example is all the heaters are switched on the total would be $2700 \mathrm{~W}$ after assuming that no window or door is open at this instant. Similarly if only two of the heaters are on then the total would be $1800 \mathrm{~W}$ only. The output variable in the graphs is the additional power required by the system. This additional power is determined by the controller.

\section{RULE BASE OF FUZZY CONTROLLER USING}

\section{LABVIEW 2009}

For our system we developed 150 rules and few of which are as follows:

- If outside temp is "very cold" AND medium term tendency is "neg big" AND short term tendency is "neg big" THEN additional input power is "neg big".

- If outside temp is "cold" AND medium term tendency is "big" AND short term tendency is "big" THEN additional input power is "big".

- If outside temp is "warm" AND medium term tendency is "zero" AND short term tendency is "very low pos" THEN additional Input power is "very low pos".

To develop and optimize such a large system efficiently, Labview software was used.

LabVIEW has several additional modules and Toolkits for Control and Simulation purposes, e.g., "LabVIEW Control Design and Simulation Module", "LabVIEW PID and Fuzzy Logic Toolkit", "LabVIEW System Identification Toolkit" and "LabVIEW Simulation Interface Toolkit". LabVIEW MathScript is also useful for Control Design and Simulation.

- LabVIEW Control Design and Simulation Module

- LabVIEW PID and Fuzzy Logic Toolkit

- LabVIEW System Identification Toolkit

- LabVIEW Simulation Interface Toolkit

All VIs related to these modules and toolkits are placed in the Control Design and Simulation Toolkit:

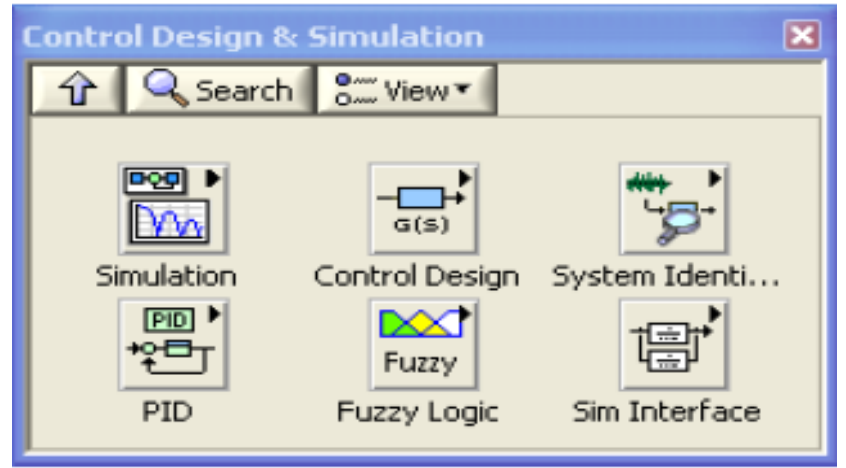

\section{Control Design Tool}

Select Tools » Control design and Simulation » Fuzzy System Designer to launch the Fuzzy System Designer. The first step in designing a fuzzy system with the Fuzzy System Designer is to create the input and output linguistic variables for the system.

First the limits for the variable are entered. The range is then divided into various linguistic labels. The labels can be described by various geometric shapes like triangle, trapezoid, Gaussian curves etc. The shapes are drawn by mentioning the limits for the curve. Thus the input and output variables are plotted.

The next step is to define the relation between the input and output variables. The rule base is defined by clicking on the rules tab. In this tab the required input variables and output are selected. Each antecedent is connected by using a suitable connective and the suitable consequent implicant is chosen.

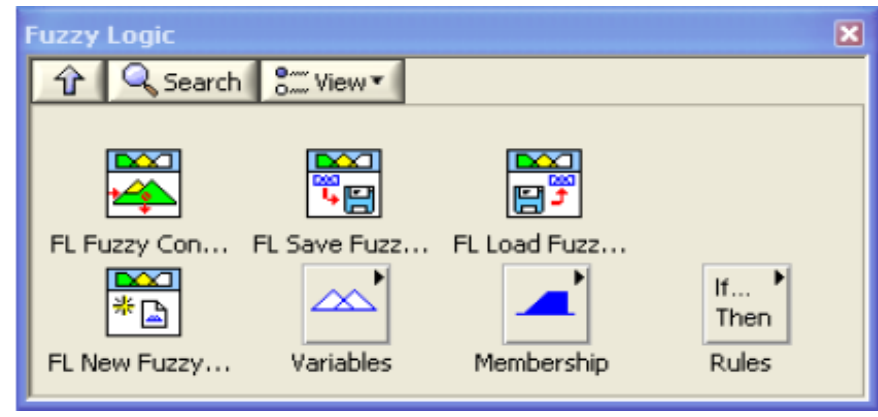

Fuzzy Designer Tool

Also the defuzzification method is selected. There is also a provision to auto generating the rule base. This method disadvantageous if all possible combinations of the input and output is required. Finally by using the test system the defuzzified value is checked for accuracy. If needed the input and output variables can be altered to achieve accuracy. Finally once the output has been obtained the controller is saved. This file is loaded into the program for processing of the input and obtaining the output. 


\section{TEST SYSTEM}

The test system of the LAB VIEW is used to test the relationship between the input and output values of a fuzzy system in order to validate the rule base of the fuzzy system. In the test system the values of the input variables are entered manually. The controller then calculates the corresponding weights of the inputs. Based on the antecedent connective and consequent implication the weight of the output is found out. The test system also displays the rules invoked.

Based on the defuzzification method used the output variable is obtained as a crisp value. The fuzzy controller then can be tuned to obtain the output variable. The test system gives an option to select the plot variables. Based on these the selection the input/ output relationship is mapped.

In our project the test system window allows us to test our fuzzy controller. It calculates the additional input power to be supplied based on the values of outside temperature, medium term tendency and short term tendency.

It calculated the weights of the inputs and calculated the weight of the output variable. This value was defuzzified to give the crisp value. The test system also displayed the rules fired correspondingly. It plotted the relationship between outside temperature (on the $\mathrm{x}$ axis), medium term tendency (on the $\mathrm{y}$ axis) and additional input power (on the $\mathrm{z}$ axis).

\section{CONCLUSIONS}

The purpose of the project was to develop an Intelligent Home Heating System which adapts itself to customer's changing heating demands over the year.

The Heating system takes into account the number of heaters turned on in every room. It also takes into account the number of doors and window. To design the system, fuzzy logic was used. The fuzzy logic controller was designed and implemented using LabView 2009. The system designed provides improved comfort, happens to be less complex as there is no mathematical modeling used and has completely eliminated the use of sensors.

The system designed recognizes the month, the number of heaters turned on, windows and doors opened. Based on these values the additional input power required is calculated. The power developed is classified as high, medium and low. The power required is represented graphically.

\section{REFERENCES}

[1] "Fuzzy logic with engineering applications" $1^{\text {st }}$ edition by Timothy J. Ross, University of New Mexico, USA.

[2] "Customer-Adaptive Fuzzy Control Of Home Heating System" -C. v. Altrock, Inform Software Corp., 1840 Oak Avenue, Evanston, IL 60201, H.-0. Arend, Viessmann Werke
GmbH \& Co., D-3559 Allendorf(Eder) B. Krause and C. Steffens, INFORM GmbH, Pascalstrasse 23, D-52076 Aachen [3] "An Occupant Dependent, Self - Optimizing HomeAutomation Syste m Using Neuro-Fuzzy Control" - Markus NEBENFUHR, Gerhard H. SCHILDT and Harald J.ZAINZINGER Interuniversitary Center for CIM Institute of Automation, Vienna University of Technology,Vienna Austria [4] http://www.ni.com/labview/

[5] http://www.ni.com/

[6] http://jki.net/vipm

[7] http://www.ieee.org/index.html

[8] http://www.isa.org/

[9] http://ivifoundation.org/ 